\title{
Menstrual Abnormalities and Associated Hormone Profile In Chronic Kidney Disease Stage 5 Patients
}

\author{
M JABIN $^{\mathrm{a}}$, MN CHOWDHURY ${ }^{\mathrm{b}}$, MN ISLAM $^{\mathrm{c}}$, MEU KHAN $^{\mathrm{d}}$
}

\begin{abstract}
:
Background: Although menstrual abnormalities and associated hormonal dysregulations are very common in the reproductive age group of Chronic Kidney Disease (CKD) patients, this remains a neglected area. This field had been poorly explored in last ten years worldwide and a few research regarding this area in Bangladesh as well.

Aim: To evaluate menstrual abnormalities occurring in CKD stage 5 (CKD5)patients undergoing twice-weekly and thriceweekly maintenance hemodialysis (HD) also in non-dialytic CKD5 patients and to provide more detail information on hormone profile (FSH, LH, Prolactin, Estradiol) of these patients.

Materials and method: This obsevational study was conducted in the Department of Nephrology, DMCH, the sample population was also collected from BSMMU and NIKDU from April 2017 to March 2018. A total of 51 CKD stage 5 patients were enrolled in this study. Among them, 34 patients were dialytic (17 of them were taking twice weekly HD and 17 of them were taking thrice weekly HD) and 17 non-dialytic patients at reproductive age were evaluated. Detailed menstrual histories, thorough clinical examinations as well as investigations were done in all the patients. Serum FSH, LH, Estradiol, and Prolactin were evaluated using chemiluminescence immunoassay in the Department of Microbiology of BSMMU. Statistical analysis of the study was done by SPSS-24. The confidence interval was considered at $95 \%$ level. P-value $<0.05$ was considered statistically significant.
\end{abstract}

Introduction

Chronic kidney disease (CKD) is the most important health concern worldwide, with a high prevalence and

a. Dr. Masrura Jabin, Assistant Professor in Department of Medicine, Nephrology, Gonoshasthaya SV Medical College and Hospital, Bangladesh.

b. Prof. Md. Nizamuddin Chowdhury, Professor, Department of Nephrology, BRB Hospitals Limited, Bangladesh.

c. Prof. Md. Nazrul Islam, Professor of Department of Nephrology, Dhaka Medical College Hospital, Bangladesh.

d. Dr. Mohammad Ehasun Uddin Khan, Assistant Professor in Department of Nephrology. Dhaka Medical College Hospital, Bangladesh.

Address of Correspondence: Masrura Jabin, Assistant Professor in Department of Medicine and Nephrology, Gonoshasthaya SV Medical College and Hospital, Bangladesh. Mobile: 01712253333, E-mail: drmasrura@gmail.com

Received: 09 May, 2021

Accepted: 18 Sept., 2021
Results: $100 \%$ of non-dialytic CKD5 women had menstrual disorders (72\% of patients had secondary amenorrhea, $18 \%$ had oligomenorrhoea and 10\% had menometrorrhagia). And $73.52 \%$ of patients in the HD group had menstrual disorders (29\% patients had regular menstruation, $28.5 \%$ had secondary amenorrhea, $23.5 \%$ had oligomenorrhoea and 19\% had menometrorrhagia). With continuation of HD amenorrhea disappeared in $43 \%$ of patients in the thrice-weekly HD group, also $22.22 \%$ patients in the twice-weekly HD group regained menstruation. Serum LH and prolactin levels were significantly higher in the non-HD group compared to the HD group $(p<0.05)$. Estradiol levels were also higher in HD patients than the non-HD patients. LH and Prolactin levels were also higher in the twice-weekly HD group compared to the thrice-weekly HD group. In the secondary amenorrheic group, serum FSH, $L H$, Prolactin levels were significantly higher than the other groups having menstrual disorder $(p<0.05)$.

Conclusion: Menstrual abnormalities and associated hormonal dysregulations were significantly lower in thriceweekly HD patients compared to the twice-weekly HD patients and significantly lower in twice-weekly HD patients compared to the non-dialytic CKD5 patients. Besides, it is suggested that long-duration dialysis might improve menstrual disorders in such patients as prolactin, LH levels gradually decreased with longer duration of dialysis.

Keywords: Chronic kidney disease, menstrual abnormalities, hormone profiles

(J Bangladesh Coll Phys Surg 2022; 40: 45-51) DOI: https://doi.org/10.3329/jbcps.v40i1.57057

incidence [1].CKD in women is often accompanied by menstrual and fertility disorders as a consequence of kidney-mediated endocrine disturbances[2].The reproductive system disorders are commonly encountered in adults of both sexes with End-stage renal disease(ESRD). The majority of premenopausal women undergoing dialysis therapy experience anovulation, abnormal menstrual cycles, often associated with secondary amenorrhoea, loss of libido. ${ }^{3,4}$

Gonadotropin-releasing hormone $(\mathrm{GnRH})$ secretion from the hypothalamus is pivotal to the regulation of reproductive physiology. The gonadotropes respond to $\mathrm{GnRH}$ pulses by releasing the gonadotropins, folliclestimulating hormone (FSH) and luteinizing hormone (LH), which stimulate folliculogenesis and hormone secretion from the ovaries. ${ }^{5}$ 
As CKD progresses, the tonic release of GnRH regulating the basal secretion of the LH and FSH appears to remain normal, but there is loss of the normal cyclic release of $\mathrm{GnRH}$ by the hypothalamus resulting in impaired ovulation. So there is inhibition of high estradiol-mediated pulsatile secretion of GnRH due to CKD. Why cyclic release of GnRH is compromised in the setting of CKD is unclear, but hyperprolactinemia and high levels of GnRH and LH caused by reduced clearance have been implicated .,6

Menstrual disturbances, especially secondary amenorrhea, are commonly seen among women of reproductive age group with End stage renal disease (ESRD). It is postulated that elevated serum prolactin (PRL) levels may partly contribute to menstrual disturbance. The mechanisms for hyperprolactinemia in CKD patients are not well defined, but a possible cause could be an increased autonomous production rate of PRL and a decreased metabolic clearance rate also play a role[2,7].As a result of increased prolactin levels, normal cyclic GnRH secretion decreases, resulting in the loss of pulsatile LH and FSH release, ultimately leading to the decline and eventual absence of estradiol release and an ensuing lack of progestational changes in the endometrium. 8,9

Besides, while amenorrhea was detected in women with high prolactin, regular menstruation was reported in women with the lowest level of prolactin The most considerable cause of these menstrual abnormalities is the increment of prolactin levels and LH levels related to impaired hypothalamic-pituitary-ovarian (H-P-O) axis . 9,10

The menstrual cycle typically remains irregular with scanty flow at the beginning of maintenance dialysis,although normal menstrual cycle is restored in some woman and ovulation might be seen with continuation of HD for longer period. ${ }^{6}$

Hormonal disturbances can be corrected by normalization of the serum PRL level through improving renal replacement therapy(RRT). Treatment with peritoneal or conventional HD does not restore H-P-O function; however, treatment with increased intensity of $\mathrm{HD}^{11}$ and kidney transplantation. ${ }^{12,13}$ often restores menses and fertility. An important reason for this condition in these cases is the long-term HD that all patients undergo and sufficient time for improvement of blood parameters. ${ }^{6}$ Prolactin levels gradually decreased with longer duration of HD. In uraemic women, menses may return after a variable length of HD treatment, but their cycles may still be irregular in duration or amount of menstrual flow. ${ }^{8}$

Thus, the role and effects of RRT on menstrual cycle remodeling in reproductive women with ESRD is still controversial. Hence, the purpose of this study was to evaluate menstrual abnormalities occurring in CKD stage 5 patients undergoing twice-weekly and thriceweekly maintenance $\mathrm{HD}$ also in non-dialytic CKD stage 5 patients and to provide more detail information on hormone profile (FSH, LH, Prolactin, Estradiol) of these patients.

\section{Materials and Methods:}

This observational study was conducted in the Department of Nephrology, Dhaka Medical College Hospital. As female CKD patients in reproductive age groups are few in numbers so patients were also collected from Bangabandhu Sheikh Mujib Medical University (BSMMU) as well as the National Institute of Kidney Diseases and Urology (NIKDU). Study period from April 2017 to March 2018. Inclusion criteria was :females in the reproductive age group (15-45 years irrespective of menstrual status) CKD stage 5 non-dialytic patients and patients getting maintenance $\mathrm{HD}$ twice or thrice per week for at least 06 months. Patients who had any gynaecological operation eg. hystrectomy and or oophorectomy, any cranial operation , any known gynaecological disease and who use hormonal contraception were excluded from the study. Patients were divided into three groups. Group 1: ESRD patients on twice-weekly HD, $\mathrm{n}=17$; Group 2: ESRD patients on thriceweekly HD, $n=17$; Group 3: CKD5 patients who are not getting HD; CKD5ND, n= 17. Detailed menstrual history before and after the HD were obtained, various demographic characteristics: age, body mass index (BMI), gravidity, parity, abortion, curettage, whether they had amenorrhea before or after the dialysis, duration of amenorrhea were recorded. Patients who previously had periods but had the absence of menstrual periods for a time period of more than six months was labeled as secondary amenorrhoea, who had period duration more than 35 days labeled as oligomenorrhoea, and who had excessive and prolonged uterine bleeding occurring at irregular and/or frequent intervals was labeled as menometrorrhagia. A thorough clinical examination was performed for all patients. Venous blood was obtained from the HD and non-HD patients, who are menstruating on the 5 th to 8 th day of menstruation (if they would have 
dialysis on the 5th day of the menstrual cycle just before the dialysis).For the non-menstruating women, the venous blood samples were collected on the day of their enrollment to this study. Serum FSH, LH, Estradiol, and Prolactin were evaluated using chemiluminescence immunoassay in the Department of Microbiology of BSMMU. Appropriate data was collected by using a preformed data collection sheet. Statistical analysis of the study was done by computer software device as the Statistical Package for Social Science (SPSS-24). The results are presented in tables, figures, and diagrams. Categorical data are presented as frequency \& percentage and numerical data as mean \& standard deviation. The confidence interval was considered at a $95 \%$ level. A pvalue of $<0.05$ is considered statistically significant.

\section{Results:}

Demographic data and comparisons of 34 patients taking hemodialysis and 17 non-dialytic CKD5 patients, a total of 51 patients are shown in Table I.
Mean and Standard Deviation was done to measure the level of significance. The mean age of the hemodialytic patients was $32.06 \pm 7.62$ years and the CKD5ND patients was $31.17 \pm 6.43$ years. The mean BMI of the hemodialytic patients was $24.73 \pm 3.42 \mathrm{~kg} / \mathrm{m} 2$ and the CKD5ND patients were $26.35 \pm 2.43 \mathrm{~kg} / \mathrm{m} 2$.

There was not any statistically significant difference ( $p>0.05$ ) between the patients in terms of age, BMI, gravidity, parity, previous abortions, and curettage.

Figure 1 shows Secondary Amenorrhea found in 43\% of patients, oligomenorrhea in $21.35 \%$ patients, regular menstruation in $18 \%$ of patients, and $17.60 \%$ menometrorrhagia

The frequency of menstrual disorders in hemodialytic and non-hemodialytic groups are compared below in figure 2 .

In figure 2: Among the thrice-weekly HD group, $38 \%$ of the patients had regular menstruation, $30 \%$ of them had

Table-I

\begin{tabular}{|c|c|c|c|c|c|}
\hline \multicolumn{6}{|c|}{ Demographic data of hemodialysis patients and the non-hemodialysis patients } \\
\hline & \multicolumn{2}{|c|}{ Hemodialysis Patients } & \multicolumn{2}{|c|}{$\mathrm{CKD}_{5 \mathrm{ND}}$ Patients } & \multirow[t]{2}{*}{ p-value } \\
\hline & Mean & $\pm \mathrm{SD}$ & Mean & $\pm \mathrm{SD}$ & \\
\hline Age & 32.06 & 7.62 & 31.17 & 6.43 & 0.378 \\
\hline \multirow[t]{2}{*}{ BMI } & 24.73 & 3.42 & 26.35 & 2.43 & 0.069 \\
\hline & Median & $(\operatorname{Min}-\mathrm{Max})$ & Median & $(\operatorname{Min}-\operatorname{Max})$ & \\
\hline Gravidity & 1.0 & $(0.0-4.0)$ & 2.0 & $(0.0-5.0)$ & 0.912 \\
\hline Parity & 1.0 & $(0.0-4.0)$ & 1.0 & $(0.0-4.0)$ & 0.834 \\
\hline Abortion & 0.0 & $(0.0-2.0)$ & 0.0 & $(0.0-2.0)$ & 0.112 \\
\hline Curettage & 0.0 & $(0.0-2.0)$ & 0.0 & $(0.0-3.0)$ & 0.088 \\
\hline
\end{tabular}

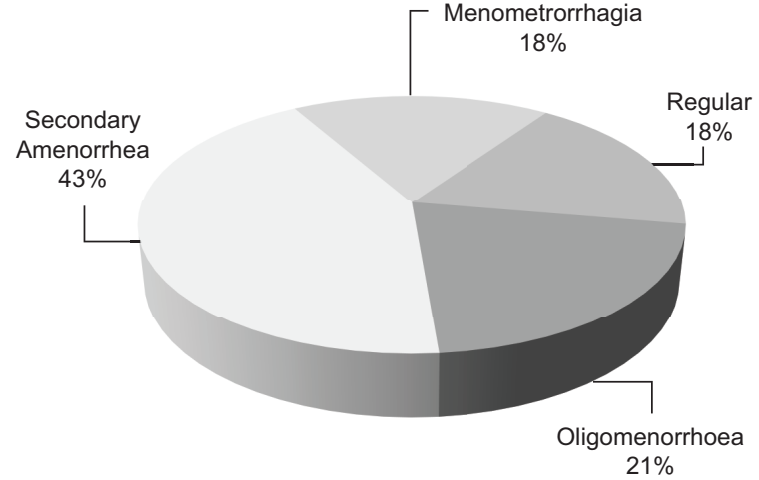

Fig.-1: Menstrual disorders in the CKD stage 5 patients.

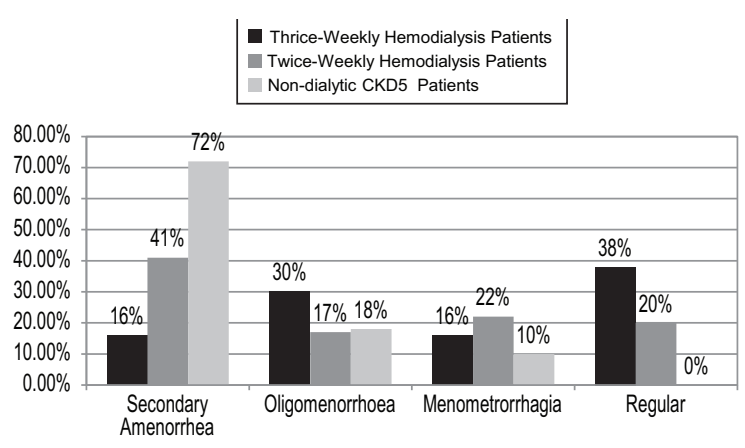

Fig.-2: Menstrual disorders of the three groups. 
oligomenorrhea, 16\% had secondary amenorrhea, and $16 \%$ had menometrorrhagia. Among the twice-weekly HD group, $20 \%$ of the patients had regular menstruation, $17 \%$ of them had oligomenorrhea, $41 \%$ had secondary amenorrhea, and 22\% had menometrorrhagia. Among the CKD5ND group, $72 \%$ of patients had secondary amenorrhea, 18\% had oligomenorrhoea and $10 \%$ had menometrorrhagia.

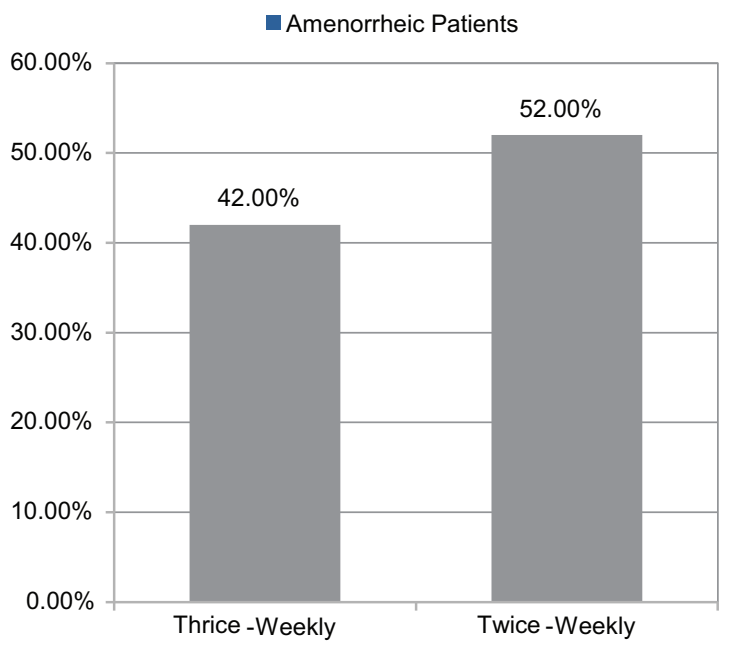

Fig.-3: Comparison of patients developing secondary amenorrhea at the beginning of HD. At the starting of $H D, 42 \%$ HD patients developed amenorrhea in the thrice-weekly HD group and 52\% in the twice-weekly HD group.

Table II shows :LH and Prolactin levels were higher in the twice-weekly HD group compared to the thrice-weekly HD group, which was statistically significant $(\mathrm{p}<0.05)$. In Table III:High levels of LH, Prolactin were found in the non-dialytic group compared to the HD group, and higher estradiol levels were seen in HD groups which is

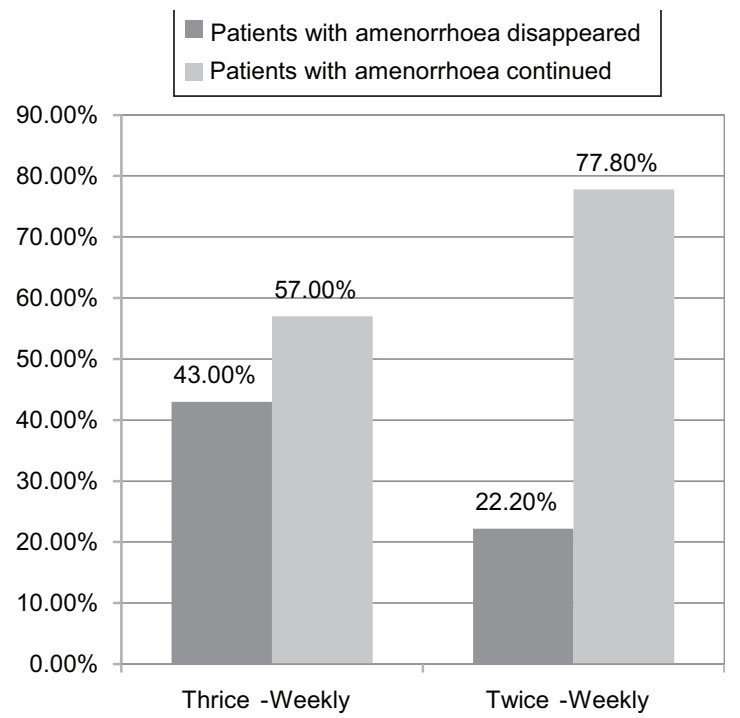

Fig.-4: Comparison of patients with amenorrhea disappeared and patients with amenorrhea continued. With subsequent HD treatment amenorrhea disappeared in $43 \%$ of patients in the thrice -weekly HD group and $22.22 \%$ patients in twice weekly HD group.

statistically significant $(\mathrm{p}<0.05)$. Despite the high level of Prolactin in the non-dialytic group, galactorrhoea was detected in only 3 patients $(13 \%)$ as well as hirsutism in 1 patient $(4.5 \%)$.

Table IV :After the patients were divided into 4 groups, according to the menstrual disorder, FSH, LH, Estradiol, and Prolactin levels were compared in each group. Mean and Standard Deviation was done to measure the level of significance. The average value of Estradiol in regularly menstruating women was significantly higher compared to the other groups. In the secondary amenorrheic group, serum FSH, LH, Prolactin levels were significantly higher than the other groups $(\mathrm{p}<0.05)$.

Table-II

Comparison of hormonal values between twice-weekly and thrice-weekly HD groups

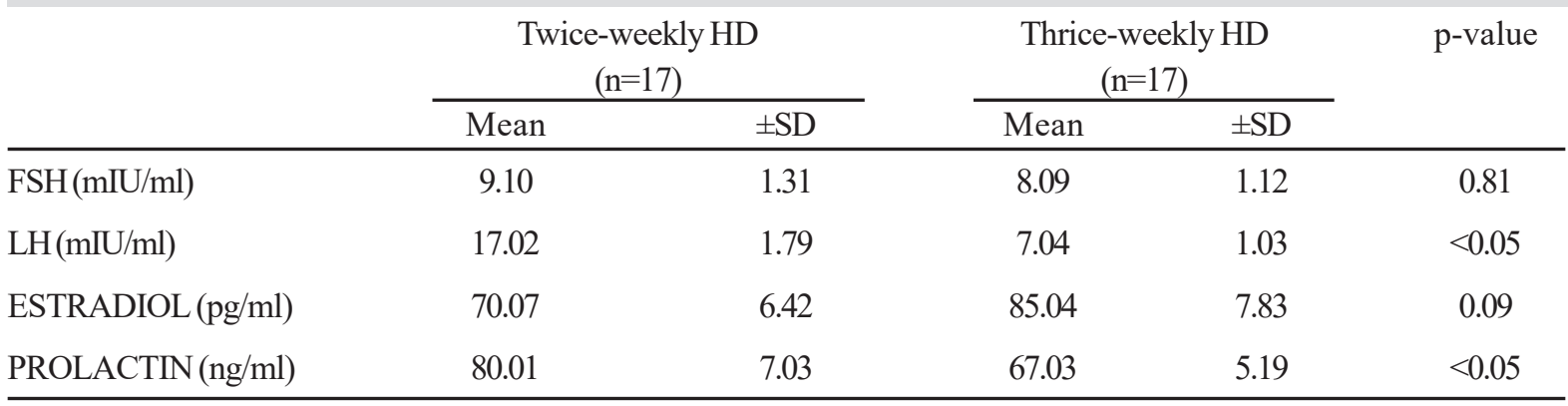


Table-III

\begin{tabular}{|c|c|c|c|c|c|}
\hline \multicolumn{6}{|c|}{ Comparison of hormonal values between CKD5ND and HD patients } \\
\hline & \multicolumn{2}{|c|}{$\begin{array}{l}\text { HD Patients } \\
\quad(\mathrm{n}=34)\end{array}$} & \multicolumn{2}{|c|}{$\begin{array}{c}\mathrm{CKD}_{5 \mathrm{ND}} \text { Patients } \\
(\mathrm{n}=17)\end{array}$} & \multirow[t]{2}{*}{ p-value } \\
\hline & Mean. & $\pm \mathrm{SD}$ & Mean. & $\pm \mathrm{SD}$ & \\
\hline$\overline{\mathrm{FSH}}(\mathrm{mIU} / \mathrm{ml})$ & 8.59 & 1.21 & 10.09 & 1.93 & 0.546 \\
\hline LH(mIU/ml) & 9.03 & 1.41 & 18.99 & 3.76 & $<0.01$ \\
\hline ESTRADIOL (pg/ml) & 73.50 & 7.12 & 32.7 & 5.39 & $<0.05$ \\
\hline PROLACTIN (ng/ml) & 77.55 & 6.11 & 142.3 & 8.02 & $<0.01$ \\
\hline
\end{tabular}

Table-IV

Hormone profile and their relationship with menstrual disorders in the whole studied population

\begin{tabular}{lcccccccc} 
Hormone & \multicolumn{2}{c}{$\begin{array}{c}\text { Regular } \\
(\mathrm{n}=9)\end{array}$} & \multicolumn{2}{c}{$\begin{array}{c}\text { Oligomeno-rrhoea } \\
(\mathrm{n}=11)\end{array}$} & \multicolumn{2}{c}{$\begin{array}{c}\text { Secondary Amenorrhea } \\
(\mathrm{n}=23)\end{array}$} & \multicolumn{2}{c}{$\begin{array}{c}\text { Menometro-rrhagia } \\
(\mathrm{n}=8)\end{array}$} \\
& Mean & $\pm \mathrm{SD}$ & Mean & \pm SD & Mean & \pm SD & Mean & \pm SD \\
\hline FSH $(\mathrm{mIU} / \mathrm{ml})$ & 10.3 & 2.07 & $15.86^{*}$ & 1.14 & $33.3^{* *}$ & 4.21 & $17.6^{*}$ & 1.15 \\
LH $(\mathrm{mIU} / \mathrm{ml})$ & 7.49 & 1.76 & $9.08^{*}$ & 2.13 & $39.98^{* * * *}$ & 5.13 & $11.5^{*}$ & 0.92 \\
ESTRADIOL $(\mathrm{pg} / \mathrm{ml})$ & $117.66^{* * *}$ & 4.71 & 85.9 & 3.05 & 44.7 & 6.18 & 5.6 & 1.07 \\
PROLACTIN $(\mathrm{ng} / \mathrm{ml})$ & $29.16^{* *}$ & 2.81 & $57.69^{* *}$ & 5.19 & $>200^{* * * *}$ & - & $113^{* * *}$ & 4.05 \\
\hline
\end{tabular}

$* p<0.05, * * p<0.01, * * * p<0.005, * * * * p<0.001$

\section{Discussion:}

Aim of this study was to find out patterns and incidence of menstrual abnormalities occurring in CKD5 patients undergoing twice-weekly and thrice-weekly maintenance $\mathrm{HD}$ also in non-dialytic CKD5 patients and to provide more detail information on hormone profile (FSH, LH, Prolactin, Estradiol) of these patients; as this field is poorly explored in recent years worldwide and very few research work regarding this area in Bangladesh as well.

In our study, $100 \%$ of non-dialytic CKD5 women had menstrual disorders and $73.52 \%$ of patients in the HD group had menstrual disorders. Near half of them had secondary amenorrhea (42.3\%). One-third of them had oligomenorrhoea (30.92\%). And also near one-third of them had menometrorrhagia (26.78\%). Authors in [6] showed that more than two-fifth of dialysis patients with CKD had menstrual problems. Authors in [8] also showed in their study the overall prevalence of menstrual disturbances was $76.1 \%$ in patients getting HD; among them $60 \%$ patients had Amenorrhea and 17.1\% had Oligomenorrhea. Authors in ${ }^{13}$ reported that most common menstrual disorder among CKD patients on HD was secondary amenorrhoea and three quarter of dialysis patients had menstrual disorder. Authors in [14] clarified in their study that, less than two fifth of dialysis women had amenorrhoea, one third had oligomenorrhoea and one forth had regular menstruation. Authors in ${ }^{15}$ showed $60 \%$ dialysis females had irregular menstrual pattern.

In this study, it is shown that in a total of $34 \mathrm{HD}$ patients, $42 \%$ of them developed amenorrhea at the starting of dialysis in the thrice-weekly HD group and $52 \%$ of them developed amenorrhea in the twice-weekly HD group. With the subsequent HD therapy, amenorrhea disappeared in many of these patients. During the study, amenorrhea disappeared in $43 \%$ of patients in a thriceweekly HD group and $22.22 \%$ amenorrheic patients in a twice-weekly HD group regained menstruation. Similar prevalence was seen in. ${ }^{6}$ They showed in their study that $47 \%$ of them developed amenorrhoea at beginning of HD and with the subsequent HD therapy, amenorrhea disappeared in the majority of these patients. During the study, amenorrhea persisted only in $36 \%$ of these 
patients. Authors in ${ }^{15}$ showed there are significant differences in the menstrual cycle before and after undergoing $\mathrm{HD}$ in women with CKD. It has been postulated that the H-P-O axis is altered following ESRD, although the normalization of hormonal status could be influenced by longer duration HD per week to correct hormonal parameters. ${ }^{8}$

It is shown in our study that, there are higher levels of LH in non-dialytic patients compared to the HD patients $(p<0.05)$. A similar result was found in $[6,8]$. Other studies have shown that LH levels were higher in premenopausal women with CKD. ${ }^{16}$ It is postulated that both the metabolic clearance of LH decreases and production increases in CKD patients.

In this present study, even though FSH levels were higher in non-hemodialytic patient compared to HD patients but there was no statistically significant difference $(p>0.05)$. Similar result was found in studies of $[8,13]$. Estradiol levels in non-dialytic patients were detected lower than the HD patients and this difference was statistically significant $(\mathrm{p}<0.05)$. As uraemia causes loss of normal pulsatile secretion of LH and FSH which leads to decline in ovarian release of estradiol. ${ }^{17,18]}$

In our study, it is found that high levels of prolactin in $76 \%$ of patients. Besides, when the dialytic and nondialytic CKD5 patients were compared for the prolactin levels, it is seen that prolactin levels were higher in the non-hemodialytic patients. Even though prolactin levels were high in $76 \%$ of the patients, galactorrhea was found only in $13 \%$ of them. Similarly, ${ }^{6}$ showed in their study that high levels of prolactin in the $73 \%$ of patients and galactorrhea was found only in $13 \%$ of them .Authors in ${ }^{19}$ showed incidence of hyperprolactinemia can be up to $80 \%$ in patients with HD. Prolactin levels rise due to increased production and decreased clearance, which may contribute to alterations in the H-P-O axis, causing suppression of gonadotrophins release leading to irregular menstrual cycles. .Authors in ${ }^{8}$ reported, longer dialysis time per week significantly improved serum PRL removal in their patients.

In this study, when the HD and non-HD patients were divided into 4 groups as ones who had regular menstruation, amenorrhoea, oligomenorrhoea and menometrorrhagia, and compared in terms of hormone profiles, FSH and LH and prolactin were statistically higher $(\mathrm{p}<0.05)$ in patients having secondary amenorrhea compared to the other patient levels. While prolactin was lower in the regular group compared to the oligomenorrhea group and lower in the oligomenorrhea group compared to the secondary amenorrhea group this difference was statistically significant $(\mathrm{p}<0.05)$. In the secondary amenorrhea group, estradiol was lower than the other groups $(\mathrm{p}<0.05)$. This result is similar to [6]. They showed higher prolactin levels and lower estradiol levels in amenorrhea group. They also stated that irregularities increase as the prolactin levels increase. It has been shown, hyperprolactinemia is an important cause of menstrual disorders in HD patients, whereas amenorrhoea was predominantly accompanied by an abnormal H-P-O axis with the highest serum prolactin, increased LH and FSH.

The main limitation of this study is the small sample size. However, The study can be further diversified by increasing the number of hospitals from different districts of the country.

\section{Conclusion:}

From the result of this study it can be said that menstrual abnormalities and associated hormonal dysregulations were significantly lower in thrice-weekly HD patients compared to twice-weekly HD patients and significantly lower in twice-weekly HD patients compared to the nondialytic CKD5 patients. Also, it is suggested that longduration dialysis might improve menstrual disorders in such patients as prolactin and LH levels gradually decrease with longer duration of dialysis.

\section{References:}

1. Carney EF. The impact of chronic kidney disease on global health. Nature Reviews Nephrology. 2020 May; 16(5):2511 .

2. Ahmed SB, Ramesh S. Sex hormones in women with kidney disease. Nephrology Dialysis Transplantation. 2016 May;31(11):1787-95.

3. Ginsburg ES, Owen WF. Reproductive Endocrinology and Pregnancy in Women on Hemodialysis. Seminars in Dialysis. 2007 Oct;6(2):105-16.

4. Alkhunaizi A, Melamed N, Hladunewich MA. Pregnancy in advancedchronic kidney disease and end-stage renal disease. Current Opinion in Nephrology and Hypertension. 2015 May;24(3):252-9.

5. Ferin, M. The Hypothalamic-Hypophyseal-Ovarian Axis and the Menstrual Cycle [Internet]. The Global Library of Women's Medicine 2008 [cited 28 August 2018] 
6. Arikan DC, Bozkurt S, Arikan I, Turgut E. Hormone profiles and theirrelation with menstrual cycles in patients undergoing hemodialysis. Journalof Turkish Society of Obstetric and Gynecology. 2011;8(1):32-9.

7. Garg DSS, Ramalakshmi DS. Menstrual disorders in chronic kidney disease: causes and management. International Journal of Clinical Obstetrics and Gynaecology. 2020 Mar;4(2):353-8.

8. Lin CT, Liu XN, Xu HL, Sui HY. Menstrual Disturbances in Pre-menopausal Women with End-Stage Renal Disease: A Cross-Sectional Study. Medical Principles and Practice. 2016;25(3):260-5.

9. Ozmen B, Ta 'skýn S, Ozcan E, S oylemez F. Unwanted Pregnancies and Contraception in Chronic Renal Failure: A Case Report and Review of the Literature. J Turkish Society Obstet Gynecol. 2006;3(2):125-7.

10. AlFaris N, ALTamimi J, AlKehayez N, AlMushawah F, AlNaeem A, AlAmri N. Prevalence of Anemia and Associated Risk Factors Among Non-Pregnant Women in Riyadh, Saudi Arabia: A Cross-Sectional Study. International Journal of General Medicine. 2021 Mar;Volume 14:765-77.

11. Hladunewich MA, Hou S, Odutayo A, Cornelis T, Pierratos A, GoldsteinM,. Intensive Hemodialysis Associates with Improved Pregnancy Outcomes: A Canadian and United States Cohort Comparison. Journal of the American Society of Nephrology. 2014 Feb;25(5):1103-9.

12. Hou S. Pregnancy in Renal Transplant Recipients. Advances in Chronic Kidney Disease. 2013 May;20(3):253-9.

13. Filocamo MT, Zanazzi M, Marzi VL, Lombardi G, Popolo GD, ManciniG. Sexual Dysfunction in Women During
Dialysis and after Renal Transplantation. The Journal of Sexual Medicine. 2009 Nov;6(11):3125-31.

14. Lessan-Pezeshki M, Ghazizadeh S, Khatami MR, Mahdavi M, Razeghi E,Seifi S. Fertility and contraceptive issues after kidney transplantationin women. Transplantation Proceedings. 2004 Jun;36(5):1405-6.

15. Nurjanah S, Damayanti FN, Riafisari AA. Differences of Menstrual Cycle Before and After Hemodialysis in Women Suffering Chronic Kidneys. Turkish Journal of Computer and Mathematics Education (TURCOMAT). 2021;12(13):6459-65.

16. Matuszkiewicz-Rowinska J, Skorzewska K, Radowicki S, NiemczykS, Sokalski A, Przedlacki J. Endometrial morphology and pituitary-gonadal axis dysfunction in women of reproductive age undergoing chronic haemodialysis - a multicentre study. NephrologyDialysis Transplantation. 2004 Jun;19(8):2074-7.

17. Gluhovschi, Gh \& Gluhovschi, Adrian \& Anastasiu, D \& Petrica, Ligia \& Gluhovschi, Cristina \& Velciov, Silvia. (2012). Chronic kidney disease and the involvement of estrogen hormones in its pathogenesis and progression. Rom J Intern Med. 50. 135-44.

18. Rathi M, Ramachandran R. Sexual and gonadal dysfunctionin chronic kidney disease : Pathophysiology. Indian Journal of Endocrinology and Metabolism. 2012;16(2):214.

19. Serret-Montaya J, Zurita-Cruz JN, Villas iýs-Keever MA, Aguilar-Kitsu A, del Carmen Zepeda-Martinez C, CruzAnleu I. Hyperprolactinemias a prognostic factor for menstrual disorders in female adolescents with advanced chronic kidney disease. Pediatric Nephrology. 2020 Feb;35(6):1041-9. 